\title{
Design and Implementation of Automated Cart for COVID-19 Patients Treatment
}

\author{
Prakash Kanade, Fortune David, Sunay Kanade
}

\section{ABSTRACT}

With the recent changes in this world due to the pandemic of COVID-19 came the need to change in technology with medical environments. There were few robotic surgeries done in medical field, but the pandemic has put the Doctors and health care workers at risk. So there came a need for rapid change in medical environment to replace man with robots with the help of AI. In this paper a AGV also called as Automatic Guided Vehicle is designed for the benefit of health community. It can also be called as Automated Cart.

The chances of health care worker getting affected from the patient in this COVID-19 is more due to the behavior of the novel Corona Virus Spread. Hence this Automated cart is designed in this paper which moves near the patient's beds delivering medicines whenever needed in time and also collects waste from patients' bed and returns to the necessary point. It is a line follower automated cart robot it makes use of certain sensors like infrared sensors and ultrasonic sensors. These sensors are used for route mapping and obstacle detection. This robot at the time of giving medicine to the patients' bed and collecting waste, it also checks the body temperature and pulse rate of the patient and sends information to the doctor via internet. The adaptability of this robot with the patients depends on the preprogram done. A microcontroller is made use for this purpose. This automated cart can be designed and implemented with low cost and the risk of Doctors, health care workers is reduced.

Keywords: Automated Cart, COVID-19, LeenaBOT.

Published Online: September 11,2021

ISSN: $2736-5492$

DOI : 10.24018 /ejcompute.2021.1.4.13

Prakash Kanade*

Hobby Researcher in Robotics, Artificial Intelligence, IOT, USA.

(e-mail: prakashsrs@gmail.com)

Fortune David

Faculty, Facilitator, LeenaBOT Robotics LLC, USA.

(e-mail: fortunekbz@2009gmail.com)

Sunay Kanade

Student, LeenaBOT Robotics LLC, USA.

(e-mail: sunaykanade6@gmail.com)

*Corresponding Author

\section{INTRODUCTION}

The COVID-19 viral infection from corona virus was started in December 2019 at Wuhan city of China. Which later turned into a pandemic and disturbed the entire world in many ways. In this pandemic the Doctors, health care workers, ambulance drivers are the mostly affected persons. With the lack in advanced technology and the absence of Vaccination, medicine there were several infections and deaths [1].

Hence came the need of medical technology to be developed and replace man with machines. The novel Corona Virus has the capacity of spreading at a higher rate, which requires frequent sanitization [2]. The Virus stays in Air, copper, carboard, plastic, steel material for a time. The lifetime is varying in different materials as shown in the Fig1 below. If a healthy person touches the infected surface or inhales from air, depending on the immunity of Human the person gets infected [3].

In this paper Automated cart is designed to replace the Doctors, Nurses and health care workers. The automated cart (robot) does the function of moving to patients' bed, distribution of medicines, Collection of waste from dustbin and its disposal and Sesing the body temperature etc and communication with doctor via internet. The cart has a LED display to communicate with the patient. The Automated cart known as $\mathrm{AGV}$ is designed with the help of Arduino microcontroller (ATmega 328) [4]. It also makes use of sensors, Power regulators, motor drivers, mobile Application, and a display unit [5].

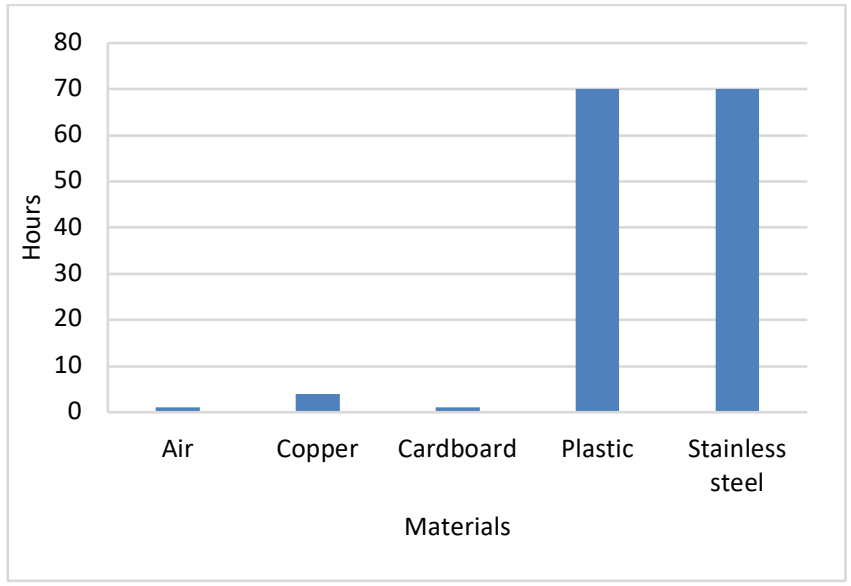

Fig. 1. Scale of Virus survival on different materials in Hours. 
Robotics mainly reduce the COVID-19 health care workers and patients' risk from getting infected at higher rate [6]. The robotic application is tested at various stages of conventional laboratory system and compared with the reality cases. This system can also be applied for automatic sanitization in the hospital, which is already present in few countries [7]. The automated cart can also be called as Aerial vehicles or drone. In developing this kind of prototype, Telemedicine, Big data, IoT, artificial intelligence, robotics plays very important role.

The prototype is designed for real time application in hospitals, which reduces the cost, increases efficiency, reduces the time of health care workers, and maintains accuracy mean while reduces the chance of Hospital authorities getting infected.

\section{DeSIGN AND IMPLEMENTATION}

The design of automated cart includes electrical and mechanical contact together to work for the complete operation. The base of the AGV is made up of plywood and steel having a carry basket on it. In the front, a LED display is made for displaying and communication. Behind LED display medical kit compartments are present. The design is implemented for three patient beds, so three medical kit compartments are present [8]. The battery used to operate this automated cart is around $12 \mathrm{~V}$ and for this purpose certain regulators are used.

\section{A. Proposed Method}

In this paper the Arduino based microcontroller takes power from the battery using a regulator circuit and controls all functions of medical kit, waste collection and displaying. Through the automatic tracking system, it also controls the operation of Motor driver circuit as shown in the Fig. 2. The sensors are also programmed in Arduino controller for Obstacle detection and Rote mapping through an interrupt command generated whenever necessary [9]. The motor controller for controlling robot is using two motors with an IC named L293d which mainly controls the right and left motors.

The guided vehicle is The Complete automatic guided vehicle is designed with certain testing and experimentation at the base level for three patient beds in a room.

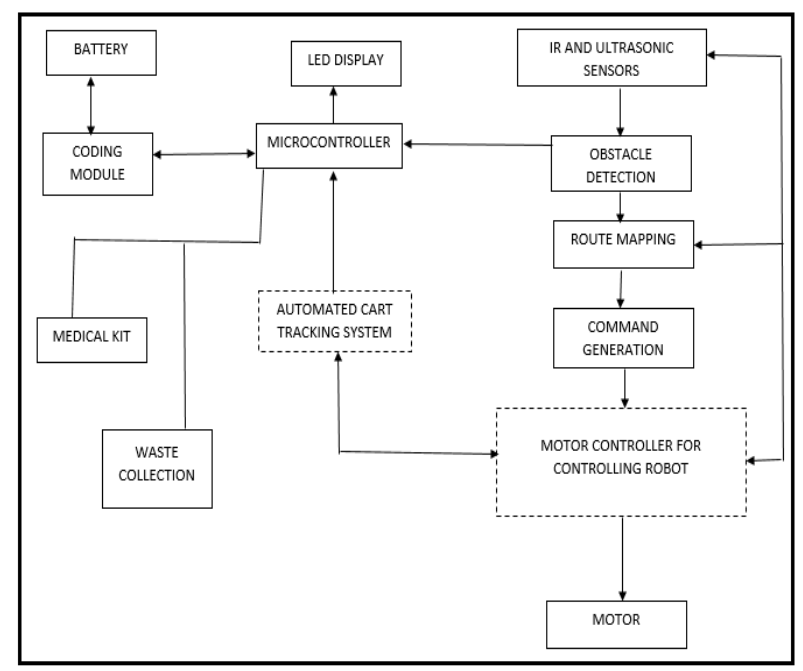

Fig. 2. Block diagram of Automatic Guided vehicle.

\section{CIRCUITRY AND PARTS}

\section{A. Main Control Unit}

Arduino based microcontroller ATmega328 is the main control unit of the automated cart. It does the storage of digital information by converting analog data to digital information from sensors and also control motor driver IC. The motor driverL293d IC in turn monitors the motors by taking turns, moving backward and forward. Based on the information received from input pins such as LOGIC LOW or LOGIC HIGH from the L293d IC the motor moves [10]. It moves to left depending on data received from left data pins and vice versa to right side from right data pins.

\section{B. Power SupplyUnit}

The power unit for the automated cart is based on switching regulator and linear regulator. The power is supplied from a battery to the regulator, which supplies to multiple output to meet the requirements of different units. The battery input voltage known as VBAT passes through the booster circuit and gets a stable output voltage VBOOST as shown in Fig. 3. It moves to a stabilizing DC circuit and drives the motor driver circuit. VBOOST regulates the $\mathrm{VCC}$ $(+5 \mathrm{~V})$ and VBUCK to supply power to the Arduino, sensors, and other units. This makes use of de voltage regulator.

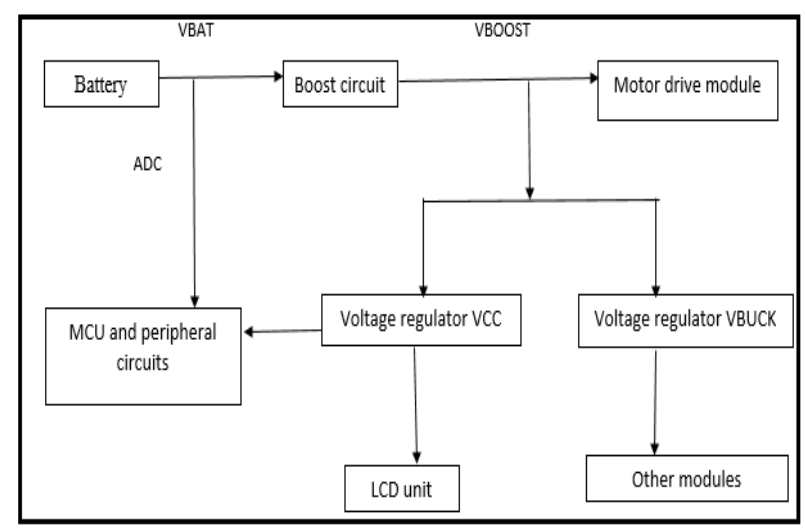

Fig. 3. Diagram of power module in the setup.

\section{Sensor Assembly}

There are two sensors in the module, one is an ultrasonic sensor placed in front of the car for obstacle detection. When the distance between the obstacle and the automated cart is less than the distance set in the program, the interrupt function of the Arduino triggers brake to the cart, either to stop or take a turn for avoiding the obstacles.

Another is an infrared sensor also known as optical guidance sensor is placed for line follower. Which makes use of a infrared LED and infrared Photodiode kept in the vehicle. It is kept beneath the vehicle and to track the tracking purpose it makes use of light and dark color lines. From the information received through the infrared light and resistance of photo diode, the voltage output changes. LED light hits the floor of facade and gets received by sensors, which detects the tint of the facade beneath it [11]. Then an information is transmitted to Arduino microcontroller to decide upon the algorithm in the program. Black color will be completely absorbed, and light color is reflected.

Here in this paper the program is written to move on black 
color identification.

\section{Navigation Module}

There are three cases of movement for the cart designed and programmed using the Arduino and motor driver IC.

- Case 1: Cart moves in forward direction when left and right side motors are on at the same time.

- Case 2: Cart moves left when right side motor is kept on and left side is turned off.

- Case 3: Cart moves right when left side motor is kept on and right side is turned off.

For this operation, the crucial element is the programming, where initially the movement control code is written for front, back, right, and left. Depending on the data inputs from the sensors the motors are driven either right or left. The flow chart of the navigation module is shown in Fig. 4.

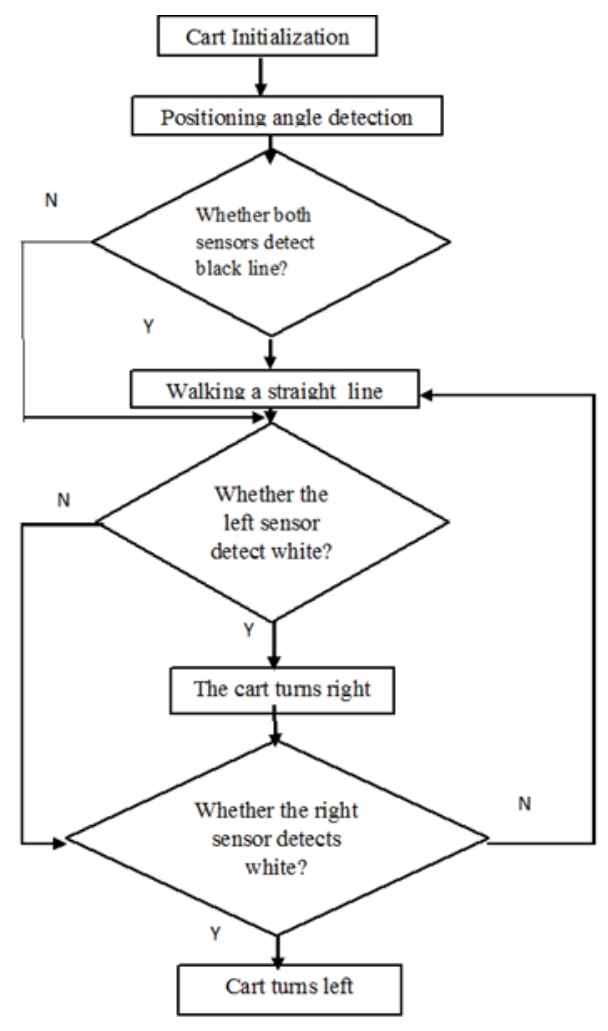

Fig. 4. Flowchart of Cart Navigation.

\section{FUnCTIONAL FEATURES}

\section{A. Medical Kit Distribution}

The primary function of the automated cart is distribution of medical kits to the right beds and right patients by collecting medicine from the available point within the hospital. This operation makes use of application developed and controlled via internet to phone or Computer. The collection of medical kits is also controlled using the same app by the nurse in the hospital. When the cart reaches the patients bed, there is a proximity sensor used to indicate the cart to stop. Later depending on the LED display board communication, the patient takes medicine from the respective kit. Only the medical kit respective to patients' bed will be opened. There are bystanders to collect medical kits.

\section{B. Waste Collection and Disposal}

In the waste collection mode of the app, the cart moves near the waste bin by line follower using black path. There will be waste bins placed at different places in the hospital. The Dc motor helps in lifting the waste bin through a cord and transferring the waste into the waste carrier in automatic cart. But complete and accurate operation of this depends on how well the system is programmed.

The maximum weight a waste carrier can handle is $6 \mathrm{KG}$. so, one weight is $6 \mathrm{KG}$ the cart moves to the dumping yard displaying carrier full in the LED. Moving to the dumping yard also follows line follower technique.

\section{Body Sensing}

To sense the temperature of the body and pulse rate there is a sensor band to be tied to the patient's hand. For this operation the LED displays the required comments and places the band into the patient's hand, senses and passes the information to the doctor. The data is stored in the cloud for further analysis in later stages.

\section{Programming Design AND COMPARISON}

The main intention of this designed AGV is the movement of cart in the right direction to the right patient and displaying along with communication via internet. The flow chart in figure 4shows the navigation module in clear to explain how exactly the program is done. The automated cart starts moving in the beginning only if both the sensors detect white using a LED and photodiode. Later depending on white or black color lines the sensor moves right or left.

The program design may be well fit to the cart, but still there is a gap in robotic field between the man and machine. So, there is a comparison Table 1 shown below collecting various data from the papers showing the difference comparison between robot and man. The work done by the workers should be so high that, it is a matter of patient's life. Whatever work the nurses used to do have to be covered completely done by robots and it should not give any pain to the patients. Sometimes depending on the age factors the usage of robots have to be controlled.

\section{RESUlTS AND DisCUSSION}

The proposed design is made to work in two modes, that is automatic mode and manual mode. The maximum weight of the waste basket near patients' bed is around $3 \mathrm{Kg}$ and that of waste carrier is $6 \mathrm{Kg}$. This Prototype is tested for three patient beds with medical box kit to serve all the three beds.

The actual AGV looks as shown in Fig. 5 below. It's with the medical kit, LED display and a basket to collect waste, along with electronic circuitry.

When the robot detects the desired patient bed, this automatic cart will stop using proximity sensor. The LED display present in the proposed model gives proper direction to the patient in the desired language to take medicines from the specific box kept to that particular patient bed. Only the medicine kit matching to that particular patient bed will open. Medical kit is operated with L293d Motor diver. Body temperature of the patient and pulse rate is sensed, and that information is also displayed in the LED for patient's information and at the same time, it is sent to the Doctor via internet. The LM35band is used for sensing temperature. The 
obstacles are detected using ultrasonic sensors and then the cart moves automatically through the route mapping provided. The route (line follower) is sensed with Infrared sensors. DC motors are used for line follower navigation. Line follower of the vehicle will be the same for waste collection from the basket [12]. Here also a DC motor is used to transfer the basket waste to waste carrier.

\begin{tabular}{cccc}
\multicolumn{4}{c}{ TABLE I: COMPARISON OF PERFORMANCE BETWEEN HUMAN AND ROBOT } \\
\hline No & Parameters & Human & Robot \\
\hline 1 & Stress sustainability & Low & High \\
2 & Attention & Varying & Constant \\
3 & Accuracy & Simulated to & Depends on \\
& human scale & programming \\
4 & Power uses & Brain power & 65 watts \\
5 & Social Skills & Excellent & Average \\
6 & Coordination & Depends on body & High Precision \\
7 & Adaptive & Very high & Design dependent \\
8 & Technical Specialty & Depends on & Design specialized \\
9 & Risk of infection & Training & High \\
10 & Multitasking & Depends on & In built ability \\
\hline
\end{tabular}

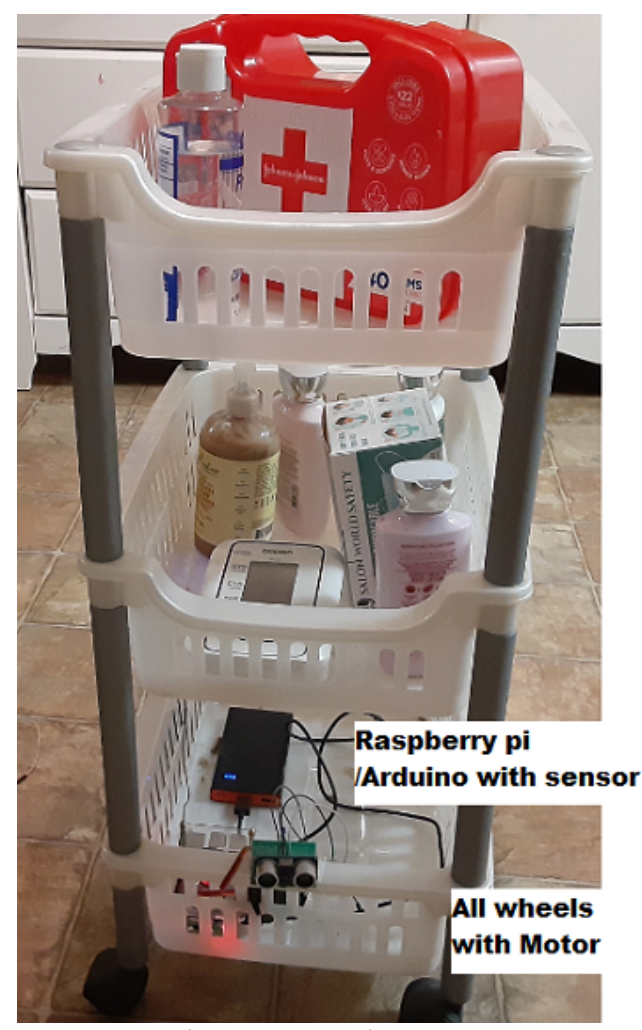

Fig. 5. Automated Cart setup.

\section{CONCLUSION}

This paper has given an insight of how exactly automated cart can be implemented in hospital environment during the pandemic situation and avoid the spread of disease. It not only replaces the burden of health care workers and Doctors; it also reduces the overall risk of getting infected in hospitals. There are certain other jobs which health care workers and doctors could do by replacement if this automated cart. Mainly this Automatic guided vehicle does three operations. First is distribution of proper Medical Kits to the patients. Second is collection of waste from the baskets in floor near the bed of patient and disposing it in right way. Third operation is the sensing of body temperature and pulse rate communicating it with the doctor. In the future this design can be extended to move in steps and automatically carry patients from ambulance to bed [13]. In other way this project can also be extended to monitor the pulse rate and give information to the doctor continuously. Only thing is robots are bit socially not responsible in skills compared to humans on programming well and making use of Artificial Intelligence it can be designed to match the social skills with human.

With the recent changes in this world due to the pandemic of COVID-19 came the need to change in technology with medical environments. There were few robotic surgeries done in medical field, but the pandemic has put the Doctors and health care workers at risk. So there came a need for rapid change in medical environment to replace man with robots with the help of AI. In this paper a AGV also called as Automatic Guided Vehicle is designed for the benefit of health community. It can also be called as Automated Cart.

The chances of health care worker getting affected from the patient in this COVID-19 is more due to the behavior of the novel Corona Virus Spread. Hence this Automated cart is designed in this paper which moves near the patient's beds delivering medicines whenever needed in time and also collects waste from patients' bed and returns to the necessary point. It is a line follower automated cart robot it makes use of certain sensors like infrared sensors and ultrasonic sensors. These sensors are used for route mapping and obstacle detection. This robot at the time of giving medicine to the patients' bed and collecting waste, it also checks the body temperature and pulse rate of the patient and sends information to the doctor via internet. The adaptability of this robot with the patients depends on the preprogram done. A microcontroller is made use for this purpose. This automated cart can be designed and implemented with low cost and the risk of Doctors, health care workers is reduced.

\section{REFERENCES}

[1] Merin Antony, Sajithkumar V.S, Megha Parameswaran, Jineeth Joseph, Christy Mary Jacob, Nithin Mathew," Design and Implementation of Automatic Guided Vehicle for Hospital Application" Proceedings of the Fifth International Conference on Communication and Electronics Systems (ICCES 2020) IEEE Conference Record \# 48766; IEEE Xplore ISBN: 978-1-7281-5371-1.

[2] Jyh-Hwa Chou, "Automatic Guided Vehicle", in Proceedings IEEE Conference on Industrial Automation and Control Emerging Technology Applications, 1995.

[3] MohdSalim Qureshi1, Pushpendra Sing, Pankaj Swarnkar, Harsh Goud, "Robotics Solutions to Combat Novel Corona Virus Disease 2019(COVID-19)", 9th International Symposium on Communications and Information Technology, pp. 1348-1349, 2009.

[4] Prakash Kanade, Prajna Alva, Sunay Kanade, Shama Ghatwal, "Automated Robot ARM using Ultrasonic Sensor in Assembly Line," International Research Journal of Engineering and Technology (IRJET), vol. 07, no. 12, pp. 615-620, 2020.

[5] Robin R. Murphy, Vignesh Babu Manjunath Gandudi Justin Adams," Applications of Robots for COVID-19 Response", arXiv:2008.06976v1 [cs.RO] 16 Aug 2020.

[6] G.-Z. Yang, B. J. Nelson, R. R. Murphy, H. Choset, H. Christensen, S. H. Collins, P. Dario, K. Goldberg, K. Ikuta, N. Jacobstein, D. Kragic, R. H. Taylor, and M. McNutt, "Combating covid-19the role of robotics in managing public health and infectious diseases," Science Robotics, vol. 5, 2020 .

[7] X. Cheng and R. Tao, "Design of Automat ic Guided Vehicles and Dunking Robot System," 2011 Third International Conference on Intelligent Human-Machine Systems and Cybernetics, Zhejiang, 2011, pp. 3-6. 
[8] Prakash Kanade, Prajna Alva, "Raspberry PI project - ultrasonic distance sensor in civil engineering," International Journal in IT \& Engineering (IJITE), vol. 8, no. 10, pp. 1-6, 2020.

[9] Shetty P, Downee S, "Modeling and simulat ion of the complete electric power $\mathrm{t}$ rain of a Hybrid Electric Vehicle" International conference on Magnetics, Machines and drives, July 24-26,2014.

[10] F. Jiménez, J. E. Naranjo "Autonomous collision avoidance system based on accurate knowledge of the vehicle surroundings," IET Intelligent Transport Systems, vol. 9, no. 1, pp. 105117, 2015.

[11] K.Y. Chee, "Control, navigat ion and collision avoidance for an unmanned aerial vehicle," In Sensors and Act uators A: Physical, vol. 190, pp. 66-76, 2013.

[12] Prakash Kanade, Sunay Kanade, "Medical Assistant Robot ARM for COVID-19 Patients Treatment - A Raspberry Pi Project," International Research Journal of Engineering and Technology (IRJET), vol. 7, no. 10, pp. 105-111, 2020.

[13] Prakash Kanade, Monis Akhtar, Fortune David, "Remote Monitoring Technology for COVID-19 Patients," European Journal of Electrical Engineering and Computer Science, vol. 5, no. 1, Pages. 44-47, 2021. 